\title{
La formación profesional de los entrepreneurs en áreas administrativas y económicas. Análisis de los emprendimientos generados en la ciudad de Quito
}

\section{The professional training of entrepreneurs in administrative and economic areas. Analysis of the ventures generated in the city of Quito}

\author{
Estefanía Salvador Tamayo
}

Fernando Hallo Alvear

Universidad Internacional del Ecuador, Ecuador

Autor para correspondencia: essalvadorta@uide.edu.ec,rohalloal@uide.edu.ec

Fecha de recepción: 07 de Agosto de 2017 - Fecha de aceptación: 30 de Septiembre de 2017

Resumen: Los negocios a nivel mundial se desarrollan con base en las actitudes y aptitudes de las personas que lo fomentan y representan uno de los impulsores de la economía a través de la generación de empleo. Se puede asegurar que un país que no haga negocios, es un país que tiende al fracaso; la relación existente entre los negocios y el desarrollo económico conlleva a un análisis específico que permita entenderlo. De acuerdo a varios autores, muchas de las pequeñas y medianas empresas PYMES - que aportan en gran medida a la economía de los países - surgen como emprendimientos que se van formando y creciendo durante el paso del tiempo. La acción de emprender incluye ciertas cualidades para la generación de nuevas ideas, productos y servicios que inspiran un sinfín de proyectos a través de dos impulsores: la necesidad y la oportunidad, buscando siempre la sostenibilidad en el tiempo mediante la innovación ligada a la satisfacción de los clientes. Con lo mencionado, en Quito se genera un alto porcentaje de oportunidades para emprender gracias al apoyo de instituciones como CONQUITO, AEI, Banco de Ideas de la SENESCYT, universidades, entre otras; esto estimula a los emprendedores a llevar adelante sus ideas y entrar en el mercado para competir. No se puede olvidar la significancia que tiene el conocimiento para los emprendimientos y cómo la educación superior genera una ventaja competitiva frente a otros emprendedores. La presente investigación busca llegar a conclusiones diagnósticas de manera cuantitativa acerca de la cantidad de emprendedores en la ciudad de Quito que han tenido su formación superior en instituciones de educación superior nacionales o extranjeras, tanto públicas como privadas. Esta, se vuelve la segunda parte de una investigación que tiene como objetivo final diagnosticar las variables que han llevado a que los emprendimientos continúen en el tiempo, identificando similitudes que puedan servir como base para los nuevos emprendedores.

Palabras Claves: emprendedores; emprendimientos; formación académica; quito

Abstract: Global business is developed based on the attitudes and aptitudes of the people who promote it and represents one of the drivers of the economy through the generation of employment. It can be assured that a country that does not do business, is a country that tends to fail; the relationship between business and economic development leads to a specific analysis that allows one to understand it. According to several authors, many small and medium-sized SMEs - which contribute greatly to the economies of the countries - appear as enterprises that are forming and 
growing over time. The action of undertaking includes certain qualities for the generation of new ideas, products and services that inspire an endless number of projects through two drivers: need and opportunity, always seeking sustainability over time through innovation linked to the satisfaction of the clients. With the aforementioned, a high percentage of opportunities for entrepreneurship is generated in Quito thanks to the support of institutions such as CONQUITO, AEI, SENESCYT Bank of Ideas, universities, among others; this stimulates the entrepreneurs to carry forward their ideas and enter the market to compete. One can not forget the significance of knowledge for entrepreneurship and how higher education generates a competitive advantage over other entrepreneurs. The present research seeks to arrive at quantitative diagnostic conclusions about the number of entrepreneurs in the city of Quito who have had their higher education in national or foreign higher education institutions, both public and private. This becomes the second part of an investigation whose final objective is to diagnose the variables that have led the ventures to continue over time, identifying similarities that can serve as a basis for new entrepreneurs.

Key words: entrepreneurs entrepreneurs academic training quito

\section{Introducción}

El emprendimiento y la innovación son términos que van de la mano y que encuentran su punto de partida en las organizaciones que inician o que tratan de despuntar en un mercado. Como lo expresa (Schnarch Kirberg, 2014), en las organizaciones, las innovaciones se catalogan según el objeto de la innovación, según el impacto de la innovación y según el efecto de la innovación. Esta afirmación conlleva a entender cómo las personas emprenden tomando como base este término.

La producción en un país es generado, entre otros factores, por los emprendimientos y éstos, a su vez, se convierten en entes generadores de empleo permitiendo la dinamización de las economías de los países. Desde el punto de vista de las personas que generan emprendimientos, (Schnarch Kirberg, 2014) establece dos tipos: los intrapreneur y los entrepreneurs. En la tabla que se presenta a continuación se establece las principales diferencias de los términos.

Tabla 1 Diferencias entre los intrapreneur y los entrepreneurs

\begin{tabular}{cc}
\hline Intrapreneur & entrepreneurs \\
\hline $\begin{array}{c}\text { Empresario dentro de la empresa } \\
\text { Produce cualquier tipo de innovación dentro de la } \\
\text { compañía }\end{array}$ & $\begin{array}{c}\text { Empresario que busca crear empresas } \\
\text { Produce cualquier tipo de innovación fuera de las } \\
\text { organizaciones }\end{array}$ \\
\hline
\end{tabular}

Nota: Diferencias establecidas de acuerdo a la postura de (Schnarch Kirberg, 2014, pág. 47)

Con lo mencionado, el entrepreneur se asocia con mucha fuerza a aquella persona que arranca un negocio desde cero, aunque teniendo claro que no siempre su quehacer resulta innovador. (Chesbrough, 2009)

Desde un punto de vista macro, (Botella \& Suárez, 2012), expresa que la innovación para el desarrollo en América Latina está por debajo de la media regional, tomando en cuenta a su población, contribución económica y nivel de desarrollo. Para (Morales \& León, 2013) las razones para innovar en América Latina son las necesidad de diferenciarse de la competencia, la presión o ambición de hacer negocios, la necesidad de generar, las ideas radicales y de mayor 
impacto, la necesidad de sobrevivir o de reinventar el modelo de negocios y el deseo de gestionar o sistematizar la innovación.

Sin embargo, es necesario determinar la importancia del emprendimiento en el Ecuador en donde, de acuerdo al reporte Global Entrepreneurship Monitor (GEM, 2016), el porcentaje de actividad emprendedora temprana (TEA) - que mide todas las iniciativas de emprendimiento existentes en el mercado y menores a tres años y medio - lidera el indicador mencionado tomando en cuenta el promedio regional de América Latina.

Ecuador forma parte de los 15 países con mayor probabilidad de iniciar emprendimientos, obtuvo el 31,8\% en el TEA que lo ubica en el segundo lugar entre 66 países participantes; siendo 2,2 veces mayor al promedio de las economías que han demostrado eficiencia al emprender un negocio. No obstante, el impacto de los emprendimientos en el Ecuador en relación a la generación de empleo es relativamente bajo, puesto que el $31 \%$ de negocios nacientes no están orientados a la generación de oportunidades laborales. (GEM, 2016)

Se puede señalar, dentro del mismo reporte, que los diferentes factores que conducen a las personas a emprender son principalmente las crisis económicas y el incremento de desempleo a nivel nacional. En este caso, el emprendimiento se considera como una de las principales alternativas para solventar los problemas microeconómicos generados por las dificultades financieras; por tanto, el emprendimiento puede generarse por necesidad o también por oportunidad.

El emprendimiento por necesidad surge generalmente de forma acelerada al implantar un negocio sin conocer el potencial de mercado que podría generarse. Por tal motivo se convierte en una aventura empresarial, pues implica riesgos económicos al considerarse una respuesta a las dificultades financieras presentadas.

Por otro lado, en el caso del emprendimiento por oportunidad, el surgimiento del negocio es construido y fundamentado a través de conocimientos previos en al área del mercado potencial. Así también se enfoca en solucionar o brindar respuestas a ciertas necesidades del mercado mediante ideas innovadoras. (Enlace PYMES, 2017)

En el país se encuentran un gran número de emprendimientos por oportunidad, en relación a las cifras de emprendedores que iniciaron su negocio por necesidad. En cuanto a cifras: el 20,8\% de la población adulta aseveró que inició un negocio por aprovechar una oportunidad, de los cuales el 22,5\% lo habría hecho por mejorar sus ingresos, el 35,2\% por obtener independencia, y el $42,3 \%$ restante por ambos motivos. Si se toma en cuenta negocios por necesidad, solamente el 8,9\% de la población mayor de 18 años, inició su negocio por este motivo.

De la misma forma, al comprar el porcentaje TEA a nivel regional, Ecuador muestra un $65,4 \%$ de emprendedores que iniciaron un negocio por oportunidad, frente a un $28 \%$ de emprendedores por necesidad. En la siguiente tabla, se detalla éste y otros indicadores que permiten relacionar los factores principales en torno al emprendimiento tanto de Ecuador como de aquellos países clasificados dentro de las economías de eficiencia. 
Tabla 2 Indicadores GEM 2016

\begin{tabular}{|c|c|c|}
\hline Indicador GEM* & Ecuador & $\begin{array}{l}\text { Economias } \\
\text { de eficiencia }\end{array}$ \\
\hline \multicolumn{3}{|c|}{ Percepciones, valores y atributos de la sociedad } \\
\hline Oportunidades percibidas & $45,5 \%$ & $42.5 \%$ \\
\hline Capacidades percibidas & $71.3 \%$ & $54.6 \%$ \\
\hline Temor al fracaso & $32.5 \%$ & $38.6 \%$ \\
\hline Emprendimiento como buena opción profesional & $59.5 \%$ & $66.9 \%$ \\
\hline Alto estatus de emprendedores exitosos & $61.1 \%$ & $66.9 \%$ \\
\hline Atención de los medios al emprendimiento & $69.5 \%$ & $61.1 \%$ \\
\hline \multicolumn{3}{|l|}{ Actividad emprendedora } \\
\hline Intención de emprendeder & $42.7 \%$ & $29.8 \%$ \\
\hline Actividad Emprendedora Temprana (TEA) & $31.8 \%$ & $14.2 \%$ \\
\hline Motivado por necesidad ( $\%$ de TEA) & $28.0 \%$ & $26.3 \%$ \\
\hline Motivado por oportunidad ( $\%$ de TEA) & $65,4 \%$ & $70.8 \%$ \\
\hline Expectativas medias/altas de crecimiento ( $\%$ de TEA) & $4.6 \%$ & $14.7 \%$ \\
\hline Actividad Emprendedora del Empleado (EEA) & $0.7 \%$ & $2.3 \%$ \\
\hline Propiedad de negocios establecidos & $14.3 \%$ & $8.6 \%$ \\
\hline Descontinuación de negocios en últimos 12 meses & $7.2 \%$ & $3.7 \%$ \\
\hline
\end{tabular}

* Definiciones de cada indicador en el Anexo A

Nota: Obtenido de (Global Entrepreneurship Monitor, 2016, pág. 10)

Al revisar los datos anteriores, se puede evidenciar que Ecuador posee un alto porcentaje de TEA en comparación con los países de América Latina. Sin embargo, es claro que existe un bajo índice motivacional, si se lo compara con países como Colombia, Perú y Chile, tal como se muestra en la siguiente tabla:

Tabla 3. Motivaciones para emprender

\begin{tabular}{|c|c|c|c|c|}
\hline & $\begin{array}{c}\text { TEA } \\
\text { (\% de población } \\
\text { 18=64 años) }\end{array}$ & $\begin{array}{c}\text { TEA } \\
\text { oportunidad } \\
\text { de mejora } \\
\text { (\% de TEA) }\end{array}$ & $\begin{array}{c}\text { TEA } \\
\text { necesidad } \\
\text { (\% de TEA) }\end{array}$ & $\begin{array}{c}\text { Índice } \\
\text { motivacional }\end{array}$ \\
\hline Ecuador & $31.8 \%$ & $34.0 \%$ & $28.0 \%$ & 1.2 \\
\hline Colombia & $27.4 \%$ & $54.6 \%$ & $13.0 \%$ & 4.2 \\
\hline Perú & $25.1 \%$ & $68.8 \%$ & $12.8 \%$ & 5.4 \\
\hline Chile & $24.2 \%$ & $63.1 \%$ & $22.7 \%$ & 2.8 \\
\hline Promedio Región & $18,8 \%$ & $49.0 \%$ & $24,6 \%$ & 2,5 \\
\hline Promedio Economias de Eficiencia & $14.2 \%$ & $46,0 \%$ & $26,3 \%$ & 2,3 \\
\hline
\end{tabular}

Nota: Obtenido de (Global Entrepreneurship Monitor, 2016, pág. 10)

La tabla anterior muestra que el promedio de la región de la TEA por oportunidad sobrepasa aproximadamente en un 50\% a la TEA por necesidad. Pero el índice motivacional es desalentador ya que el promedio regional excede al promedio del Ecuador en un $48 \%$, siendo uno de los principales propulsores para el éxito de un negocio.

Por otra parte, al desagregar este factor en las edades participantes al momento de emprender, el grupo más activo en la creación de negocios está en el rango de los 25 a 34 años de edad, con una motivación principalmente mixta, seguida muy de cerca por la motivación por 
oportunidad. Como segundo grupo, se encuentran las personas en el rango de 35 a 44 años de edad, los cuales se encuentran con motivaciones mixtas en su gran mayoría. Finalmente, el grupo entre 18 y 24 años de edad se inclina más por la motivación por oportunidad. A continuación, en la tabla 4 se presenta estos datos detallados:

Tabla 4. Motivación para emprender según edad

\begin{tabular}{|l|c|c|c|}
\hline Grupo de edad & Oportunidad de mejora & Motivación mixta & Necesidad \\
\hline $18-24$ & $21.61 \%$ & $18.24 \%$ & $16.00 \%$ \\
$25-34$ & $30.65 \%$ & $33.78 \%$ & $33.50 \%$ \\
$35-44$ & $21.11 \%$ & $29.05 \%$ & $22.50 \%$ \\
$45-54$ & $15.08 \%$ & $14.86 \%$ & $15.50 \%$ \\
$55-64$ & $11.56 \%$ & $4.05 \%$ & $12.50 \%$ \\
\hline
\end{tabular}

Nota: Obtenido de (Global Entrepreneurship Monitor, 2016, pág. 30)

Adicionalmente, es necesario mencionar el impacto que tiene la generación y desarrollo de emprendimientos en el crecimiento económico de un país. Contrario a lo que se supone, el impacto de los emprendimientos en el Ecuador es relativamente bajo, puesto que el 72,8\% de los negocios nacientes son unipersonales, y apenas el 4,6\% crean más de 10 empleos, esperando un incremento en los próximos cinco años, de apenas un 50\% de la fuerza laboral ya establecida.

Así también, al relacionar los emprendimientos con el índice motivacional, el $40 \%$ de los negocios por necesidad no esperan generar empleo en los próximos cinco años, en contraste con el 64\% de los emprendedores por oportunidad que esperan crear entre 1 y 5 empleos durante el mismo período (Global Entrepreneurship Monitor, 2016).

Otro punto importante tratado en el reporte GEM (2016), señala la falta de inversión en innovación y tecnología, lo cual ha frenado varios proyectos emprendedores con un alto componente científico y tecnológico. Además, no existe motivación por parte de los emprendedores, los cuales en su mayoría se han establecido en base a la necesidad. En este caso el crecimiento a largo plazo de los negocios es muy limitado. Este tipo de obstáculos disminuye su capacidad de producción y atractivo en el mercado (Global Entrepreneurship Monitor, 2016).

\section{Relación entre emprendimiento y formación profesional del estudiante}

Según el Banco Mundial (2016) la formación académica universitaria de los negociantes en América Latina representa el 14\%, en contraste con la educación primaria y secundaria con $69 \%$ y sin educación $17 \%$.

Una de las razones que ha fomentado la creación de negocios en el Ecuador es el desempleo al que se enfrentan los estudiantes que han concluido sus estudios de educación superior. Adicionalmente, cuando la formación profesional no ha sido adecuada y no ha generado habilidades cognoscitivas teóricas y prácticas para ser aplicadas en el mercado laboral, el encontrar un empleo es muy difícil y es entonces donde surge el emprendimiento por necesidad. (Rovayo, 2009) 
De acuerdo a datos presentados anteriormente, la edad promedio del emprendedor en Ecuador es de 36 años. En la siguiente tabla se puede observar que el promedio en años de escolaridad es 10,37 años. Por lo cual se asegura que los emprendedores no son graduados del colegio en su mayoría. Así también, se señala que al comparar el periodo 2010-2015 con el año 2016 hubo un aumento en la formación en educación superior en 2 puntos porcentuales. Este incremento se puede atar al hecho de que el gobierno ecuatoriano ha invertido una gran parte de su presupuesto en la educación; sin embargo, no se descarta otros motivos externos.

Tabla 5 Promedio factores

\begin{tabular}{|l|l|c|c|c|}
\hline \multicolumn{1}{|c|}{ Factor } & \multicolumn{1}{|c}{ Descripción } & $\begin{array}{c}\text { Promedio } \\
\text { 2010-2015 }\end{array}$ & 2015 & 2016 \\
\hline A & Apoyo financiero & 3.95 & 3.76 & 2.86 \\
B1 & Políticas gubernamentales: Políticas generales & 4.82 & 5.09 & 3.38 \\
B2 & Políticas gubernamentales: Regulación & 2.28 & 3.68 & 2.68 \\
C & Programas gubernamentales & 4.41 & 4.75 & 3.36 \\
D1 & Educación y formación; Primaria y secundaria & 4.27 & 4.21 & 3.07 \\
D2 & Educación y formación: Educación superior & 3.66 & 6.76 & 5.33 \\
E & Transferencia de I+D & 3.83 & 4.03 & 3.17 \\
F & Infraestructura comercial y profesional & 5.19 & 5.30 & 4.57 \\
G1 & Apertura del mercado interior: Dinámica & 4.22 & 4.14 & 4.54 \\
G2 & Apertura del mercado interior: Apertura & 2.75 & 4.55 & 4.21 \\
H & Acceso a infraestructura física & 7.29 & 7.99 & 6.72 \\
I & Normas sociales y culturales & 5.33 & 6.24 & 5.35 \\
\hline
\end{tabular}

Nota: Obtenido de (Global Entrepreneurship Monitor, 2016, pág. 22)

Con base en los datos anteriores, cabe mencionar que en el año 2016, entre los factores más relevantes al momento de emprender están: acceso a infraestructura física, seguido de educación superior y la infraestructura comercial y profesional a la que tienen acceso los emprendedores. Estos factores se interrelacionan tanto para iniciar un negocio como para lograr que genere un impacto positivo en la economía del país.

Posteriormente, al analizar los datos del nivel educacional de los emprendedores de la TEA, los principales actores son personas que han completado la secundaria con un 32,91\%, seguido de personas con primaria completa con un 31,99\%. Finalmente, solo el 13,53\% de los emprendedores han completado sus estudios universitarios, lo cual es alarmante si queremos asegurar una correlación positiva del nivel de educación con la motivación para emprender.

Siguiendo la misma línea, la Tabla 6 presentan los siguientes datos.

Tabla 6. Motivación por nivel educativo

\begin{tabular}{|l|r|r|r|}
\hline & $\begin{array}{c}\text { Oportunidad } \\
\text { de mejora }\end{array}$ & $\begin{array}{c}\text { Motivación } \\
\text { mixta }\end{array}$ & Necesidad \\
\hline Ninguna & $7.5 \%$ & $10.1 \%$ & $17.1 \%$ \\
Primaria Completa & $33.7 \%$ & $29.1 \%$ & $36.2 \%$ \\
Secundaria Completa & $29.6 \%$ & $32.4 \%$ & $36.7 \%$ \\
Post-Secundaria & $9.5 \%$ & $12.2 \%$ & $6.5 \%$ \\
Universidad Completa & $19.1 \%$ & $14.2 \%$ & $3.5 \%$ \\
Masterado/Doctorado & $0.5 \%$ & $2.0 \%$ & $0.0 \%$ \\
\hline
\end{tabular}

Nota: Obtenido de (Global Entrepreneurship Monitor, 2016, pág. 31) 
El motor principal para las personas con estudios de secundaria completa, es la necesidad $(36,7 \%)$, seguido de una motivación mixta. Por otro lado, quienes finalizaron los estudios de universidad se encuentran motivados en su mayoría por la oportunidad de mejora $(19,1)$ en comparación con la necesidad (3,5\%). (Global Entrepreneurship Monitor, 2016).

Adicionalmente, la siguiente tabla presenta datos según el nivel de ingresos, donde se evidencia una fuerte relación entre un mayor número de años de escolaridad con el aumento en los ingresos. De la misma forma, indica una relación positiva entre la motivación para emprender y los ingresos percibidos. La principal motivación para quienes ganan menos de $\$ 366$ fue la necesidad con un 49,30\%; conforme incrementan los ingresos, el porcentaje de motivación por necesidad disminuye y el porcentaje por oportunidad de mejora aumenta. De esta manera, el $40,63 \%$ de quienes obtienen ingresos superiores a $\$ 733$ fue motivado a emprender por oportunidad de mejora, y únicamente el $28,91 \%$ por necesidad.

Tabla 7. Estadísticas según nivel de ingresos

\begin{tabular}{|c|c|c|c|c|c|c|c|c|}
\hline & & \multicolumn{2}{|c|}{ Género } & \multirow{2}{*}{$\begin{array}{l}\text { Años de } \\
\text { escolaridad } \\
\text { promedio }\end{array}$} & \multirow{2}{*}{$\begin{array}{c}\text { Edad } \\
\text { promedio }\end{array}$} & \multicolumn{3}{|c|}{ Motivación para emprender } \\
\hline & & Hombres & Mujeres & & & $\begin{array}{l}\text { Oportunidad } \\
\text { de mejora }\end{array}$ & $\begin{array}{l}\text { Motivación } \\
\text { mixta }\end{array}$ & Necesidad \\
\hline \multirow{3}{*}{$\begin{array}{l}\text { No poseedor } \\
\text { de negocios }\end{array}$} & Menos de $\$ 366$ & $42.44 \%$ & $57.56 \%$ & 8.10 & 34.95 & & & \\
\hline & Entre $\$ 367$ y $\$ 732$ & $47.74 \%$ & $52.26 \%$ & 10.70 & 33.96 & & & \\
\hline & Más de $\$ 733$ & $57.95 \%$ & $42.05 \%$ & 13.11 & 36.17 & & & \\
\hline \multirow{3}{*}{ TEA } & Menos de $\$ 366$ & $45.58 \%$ & $54.42 \%$ & 8.38 & 35.06 & $28.87 \%$ & $21.83 \%$ & $49.30 \%$ \\
\hline & Entre $\$ 367$ y $\$ 732$ & $50.20 \%$ & $49.80 \%$ & 10.31 & 36.33 & $40.25 \%$ & $25.73 \%$ & $34.02 \%$ \\
\hline & Más de $\$ 733$ & $64.20 \%$ & $35.71 \%$ & 13.51 & 34.72 & $40.63 \%$ & $30.47 \%$ & $28.91 \%$ \\
\hline
\end{tabular}

Nota: Obtenido de (Global Entrepreneurship Monitor, 2016, pág. 32)

Por medio de estos datos se observa que la educación y el emprendimiento no siempre van de la mano; sin embargo, tiene resultados positivos si se considera el impacto que generan los emprendedores que han tenido educación superior en la creación de mayor valor agregado a la sociedad. Si bien es cierto, la mayor cantidad de emprendimientos por personas con estudios secundarios han sido generados por necesidad, el mayor porcentaje de negocios que generan ingresos menores a $\$ 366$ corresponde a este grupo, esto no representa impacto económico para el Ecuador, pues son emprendimientos unipersonales o con expectativas de crecimiento reducidas, que no esperan generar plazas de trabajo ni inversiones en investigación e innovación. (Revista M, 2015)

A su vez, la mayor cantidad de emprendimientos en el país surgen por oportunidad y los ingresos incrementan junto con los años de escolaridad. No obstante, las cifras del Ecuador en cuanto a impacto de este tipo de emprendimientos en la economía es bajo, pues muchos de los negocios no se logran consolidar en el largo plazo. El Ecuador se encuentra en el puesto 55 de 64 en aporte de innovación, y en el puesto 58 en cuanto a expectativas de empleo (GEM. Global Report, 2016, pág. 53).

Estas cifras pueden revertirse si existen mayores propuestas de innovación, de la mano con políticas públicas eficientes, en las cuales el tema de educación para el emprendimiento sea un eje fundamental que permita direccionar los emprendimientos en el Ecuador hacia el fortalecimiento de la economía (El Universo, 2017). 


\section{Metodología}

Los estudios cualitativos se fundamentan en las experiencias y en la intuición del investigador, su aplicación se da en un menor número de casos, se pueden ir enfocando en conceptos importantes durante la investigación, el aprendizaje se da a través de experiencias y puntos de vista de las personas, tal como lo expresa Hernández Sampieri, Fernández Collado, \& Baptista Lucio (2006). Para el presente trabajo investigativo se manejará un enfoque cualitativo debido a que se sustentará en datos y estudios realizados respecto a los emprendimientos generados en la ciudad de Quito por entrepreneurs que poseen formación profesional en instituciones de educación superior.

Para el trabajo de investigación se utilizó una investigación descriptiva, que como lo expresa (Bernal C. , 2010) es aquella en que se reseñan las características del objeto de estudio describiendo los aspectos más característicos, distintivos y particulares de las personas, situaciones o cosas que las hacen reconocibles.

La aplicación de esta investigación se realizará analizando 1.300 emprendimientos registrados en las bases de datos de distintas instituciones públicas y privadas relacionadas al tema. Al ser una población finita no se consideró el cálculo de la muestra. Los criterios de inclusión fueron todos los emprendimientos generados entre el año 2006 y 2016 en la ciudad de Quito.

El método deductivo utilizado parte de premisas mayores asumiendo que las conclusiones están dentro de éstas; la aplicación de este método consiste en analizar un fenómeno particular a la luz de verdades válidas universalmente, como postulados, teoremas, leyes, principios, entre otros. (Bernal C. , 2010)

\section{Resultados}

Analizando los datos obtenidos de las bases investigadas, el número de emprendimientos generados entre el 2006 y 2016 fue de 1298, siendo los últimos tres años los que presentaron un mayor número de registros, con 614, 491 y 181 respectivamente. Los datos se presentan en la Figura 1.

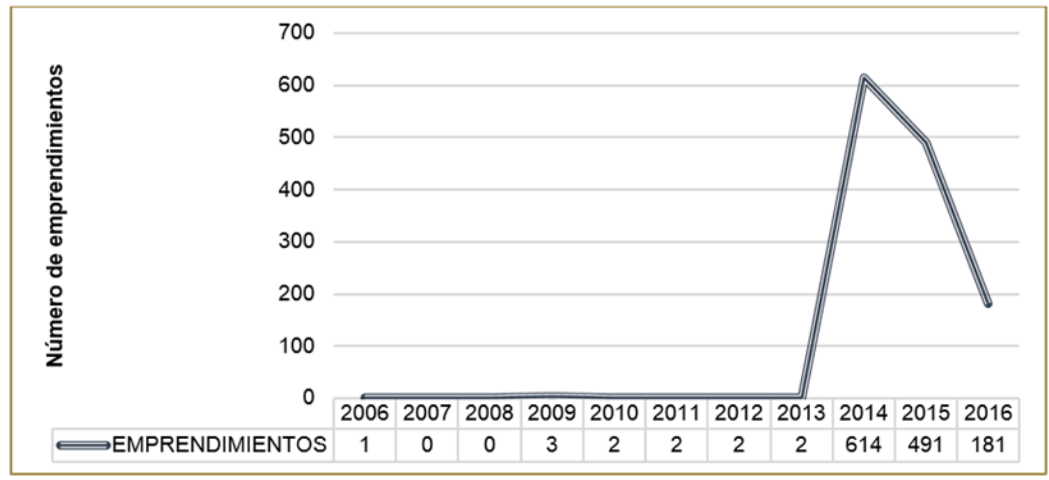

Figura 1. Emprendimientos en la Ciudad de Quito 2006-2016 
De este número de emprendimientos durante el decenio 2006 - 2016, se investigó la cantidad de entrepreneurs que obtuvieron un título profesional registrado en la Secretaría de Educación Ciencia y Tecnología SENESCYT. La importancia de revisar el registro es avalar la legalidad de la formación académica de las personas investigadas.

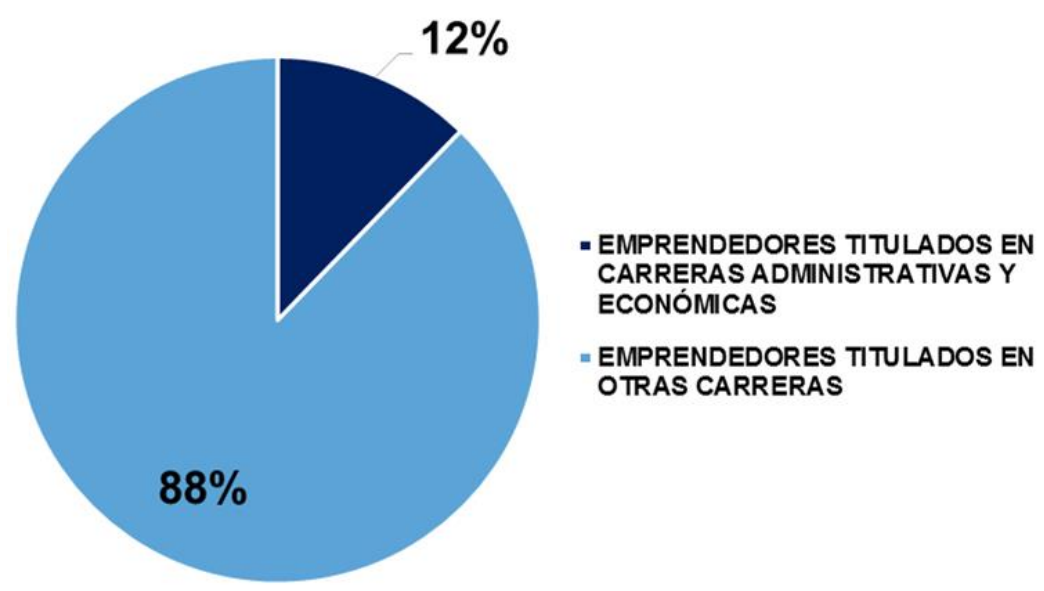

Figura 2. Titulación de los emprendedores

De la figura presentada se evidencia que únicamente el $12 \%$ de los entrepreneurs tienen una titulación profesional en carreras administrativas y económicas, siendo esta parte en donde se enfocará el estudio. Es importante mencionar que la formación en estas carreras se la tomó tanto para instituciones de educación superior nacionales como extranjeras.

Con la finalidad de observar la distribución o detalle de los títulos pertenecientes a las carreras administrativas y económicas, se unificaron el nombre de las mismas apegándose al Reglamento de Armonización dela Nomenclatura de Títulos Profesionales y Grados Académicos que confieren las Instituciones de Educación Superior del Ecuador emitido por el (CES, 2014)

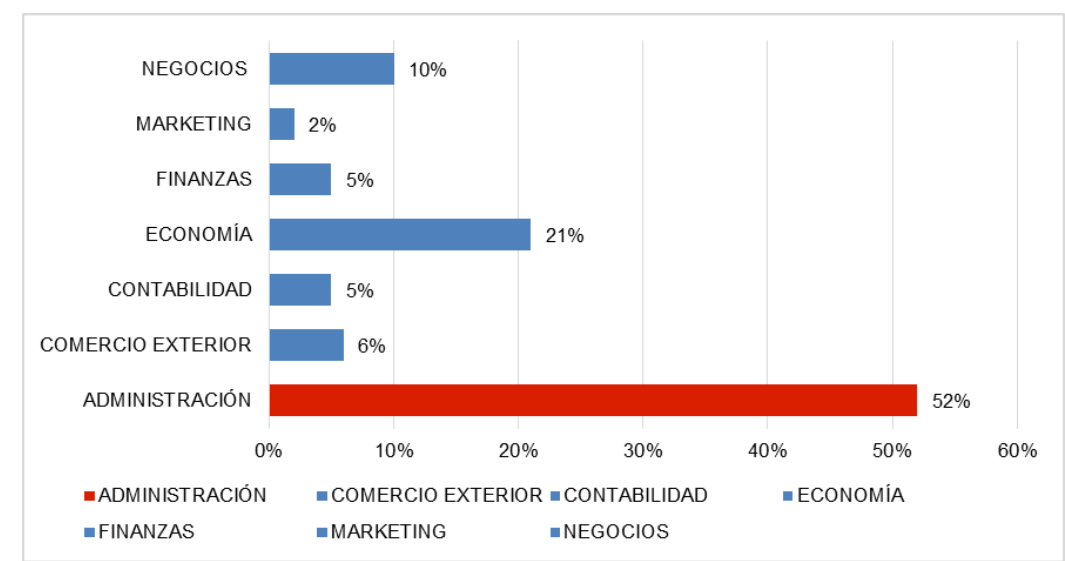

Figura 3. Detalle de los títulos de carreras administrativas y económicas obtenido por los emprendedores

Es evidente que la mayor parte de títulos obtenidos por lo entrepreneurs son relacionados a la administración de empresas, seguido bastante atrás por economía y negocios. De cierta forma, esto permite evidenciar que la formación académica en estas áreas, aparte de ser las 
preferidas por las personas que emprenden, son las más ofertadas por las instituciones de educación superior en Quito, como se lo expuso en investigaciones pasadas.

Bajo este contexto se analizó la procedencia de los títulos para conocer el porcentaje de nacionales como de extranjeros, la distribución entre públicas y privadas, y si son títulos terminales de universidades o títulos intermedios de institutos técnicos o tecnológicos, obteniendo las siguientes figuras.

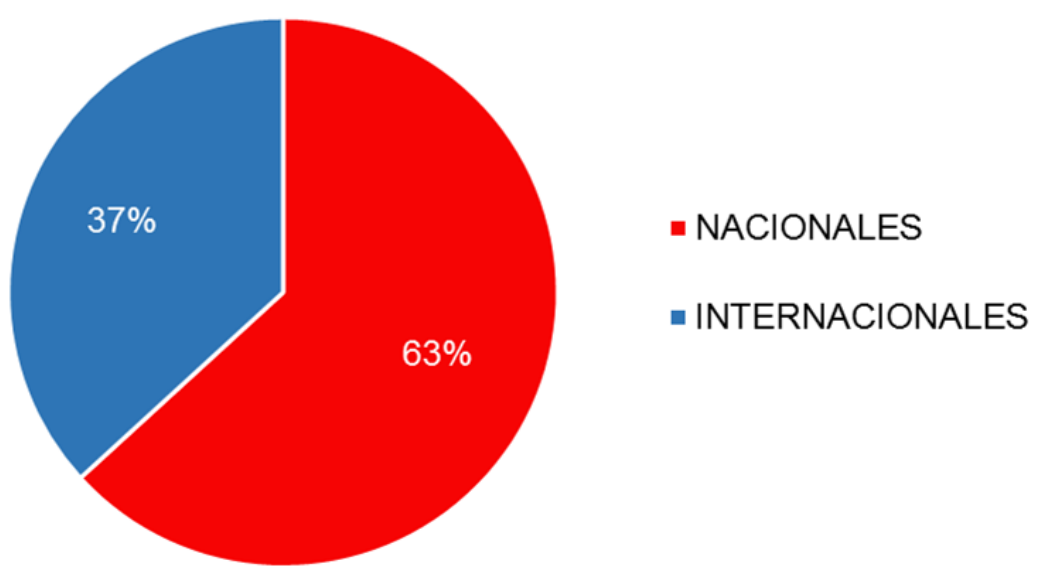

Figura 4. Procedencia de los títulos profesionales de los entrepreneurs

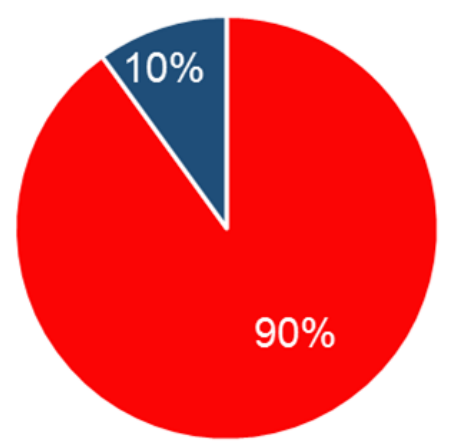

\section{- UNIVERSIDADES}

- INSTITUTOS

Figura 5. Categorización de las instituciones que emitieron los títulos profesionales a los entrepreneurs

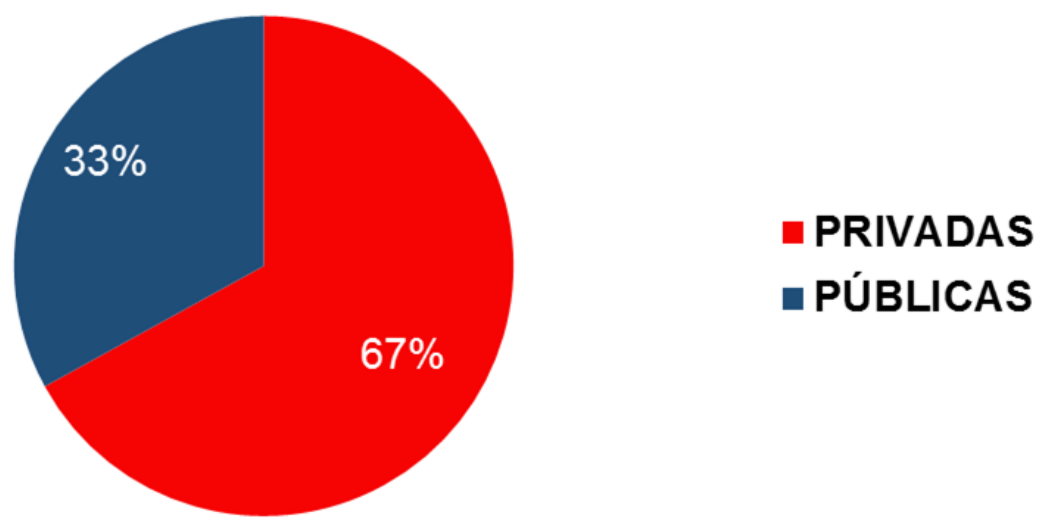

Figura 6. Tipos de instituciones que emitieron títulos profesionales a los entrepreneurs 
Con las figuras expuestas se evidencia que el mayor porcentaje de títulos que poseen los entrepreneurs son nacionales procedentes de universidades privadas. Este dato permite interpretar que las personas que emprendieron en el decenio investigado tienen una formación por la cual pagaron con sus propios recursos prefiriendo llegar a títulos terminales antes de los intermedios como técnicos o tecnólogos. Factor que será de suma importancia para investigaciones futuras en donde se pretenda interpretar el perfil del empresario emprendedor.

\section{Conclusiones}

En la ciudad de Quito, de la muestra analizada durante el período 2006 - 2016 muestra una mayor generación de emprendimientos a partir del año 2014 donde el despunte es significativo. De los 1300 emprendimientos identificados tan solo el 39\% de los entrepreneurs cuentan con título terminal de tercer nivel, independiente del área de enfoque del estudio. Por otro lado, de los 506 emprendimientos estudiados, el 52\% corresponde a títulos relacionados a carreras administrativas, en su mayoría ingenierías comerciales, seguidas muy por atrás con economía y negocios.

El 48\% de los emprendimientos incluye carreras dentro del área en estudio, como por ejemplo marketing, finanzas, negocios internacionales y economía aunque todas con menores porcentajes en comparación de administración. De las Instituciones de Educación Superior analizadas en el presente estudio, el $67 \%$ corresponde a instituciones privadas ubicadas en la capital, de las cuales el $90 \%$ representan universidades en comparación con los institutos técnicos o tecnológicos.

Es evidente que la formación profesional de los entrepreneurs en áreas administrativas y económicas en muy baja, solamente el $12 \%$ del total forman parte de ellos. Esta cifra puede representar un factor importante de análisis, puesto que puede estar atado directamente a la poca duración o tiempo de vida que tienen los emprendimientos al lanzarlos al mercado, y que según cifras del (GEM, 2016) bordean los 2 años.

Esta investigación da el punto de partida para una nueva en donde se busque identificar perfiles de los entrepreneurs que tienen formación profesional, factores de éxito que marcan la diferencia entre permanecer o desaparecer del mercado, así como también posibles variables que permitan a las instituciones formar mejores profesionales que emprendan.

\section{Bibliografía}

Alarcón Flor, R. (2009). Cómo iniciaron y se posicionaron las mejores empresas ecuatorianas. Compromiso, 8-11.

Bernal, C. (2010). Metodología de la Investigación. Bogotá: Pearson Educación.

Botella, C., \& Suárez, I. (mayo de 2012). Innovación para el desarrollo en América Latina. Una aproximación desde la cooperación internacional. Madrid: CeALCI- Fundación Carolina.

CES. (2014). Reglamento de Armonización dela Nomenclatura de Títulos Profesionales y Grados Académicos que confieren las Instituciones de Educación Superior del Ecuador. Quito. 
Chesbrough, H. W. (2009). Innovación abierta. España: Plataforma.

Christensen, C., Anthony, S., \& Roth, E. (2004). Seeing what's next: Using the theories of innovation to predict industry change. Harvard Business School Press.

Drucker, P. (1986). La innovación y el empresario innovador. Bogotá: Norma.

El Universo. (30 de abril de 2017). En Ecuador se dificulta sostener una iniciativa de emprendimiento. Obtenido de http://www.eluniverso.com/noticias/2017/04/30/nota/6159832/pais-se-dificulta-sosteneriniciativa

Enlace PYMES. (23 de 07 de 2017). Enlace PYMES. Obtenido de http://blog.enlacepymes.com/emprendedores-por-necesidad-o-por-oportunidad/

GEM. (2016). Global Entrepreneurship Monitor. ESPAE-ESPOL.

Herrera Heredia, M. (2009). Siglo XXI - La Economía del Conocimiento y la Educación. Compromiso, 35-39.

Jarrín, F. (2009). Habilidades gerenciales de las PYMES de Quito. Compromiso, 12-18.

Kastika, E. (2005). Organización innovadora. Argentina: Macchi.

Mike Herrington, P. K. (2016). GEM. Global Report. Obtenido de Ecuador: file://C:/Users/Hp/Downloads/gem-2016-2017-global-report-web-version-updated210417-1492789938.pdf

Morales, M., \& León, A. (2013). Adiós a los mitos de la innovación: Una guía práctica para innovar en América Latina. Costa Rica: Innovare.

Revista M. (2015). Educación y emprendimiento: no siempre van de la mano. Obtenido de http://mprende.co/emprendimiento/educaci\%C3\%B3n-y-emprendimiento-no-siemprevan-de-la-mano

Rovayo, G. (2009). El emprendimiento y la educación no siempre van en la misma dirección. Obtenido de http://www.usfq.edu.ec/publicaciones/polemika/Documents/polemika002/polemika002_0 14_articulo010.pdf

Schnarch Kirberg, A. (2014). Desarrollo de nuevos productos. Creatividad, innovación y marketing. McGraw-Hill.

Servicio de Rentas Internas, S. (2015). La Nueva Economía en la Nueva Constitución del Ecuador. Quito: Publingraf Industria Gráfica.

Virginia Lasio, G. C. (2016). Global Entrepreneurship Monitor. ESPAE - ESPOL. 